\title{
RAMANUJAN'S CUBIC TRANSFORMATION INEQUALITIES FOR ZERO-BALANCED HYPERGEOMETRIC FUNCTIONS
}

\author{
M.K. WANG, Y.M. CHU AND Y.P. JIANG
}

\begin{abstract}
In this paper, a generalization of Ramanujan's cubic transformation, in the form of an inequality, is proved for zero-balanced Gaussian hypergeometric function $F(a, b ; a+b ; x), a, b>0$.
\end{abstract}

1. Introduction. For real numbers $a, b$ and $c$ with $c \neq 0,-1,-2, \ldots$, the Gaussian hypergeometric function is defined by

$$
F(a, b ; c ; x)={ }_{2} F_{1}(a, b ; c ; x)=\sum_{n=0}^{\infty} \frac{(a, n)(b, n)}{(c, n)} \frac{x^{n}}{n !},
$$

for $x \in(-1,1)$, where $(a, n)$ denotes the shifted factorial function $(a, n)=a(a+1)(a+2)(a+3) \cdots(a+n-1)$ for $n=1,2, \ldots$, and $(a, 0)=1$ for $a \neq 0$. Also, $F(a, b ; c ; x)$ is called zero-balanced if $c=a+b$.

It is well known that $F(a, b ; c ; x)$ has many important applications in various fields of the mathematical and natural sciences $[4,7]$, and many classes of special function in mathematical physics are particular cases of this function [8]. For a extensive list of $F(a, b ; c ; x)$, see $[\mathbf{1}, \mathbf{2}, \mathbf{3}, \mathbf{9}]$.

As a special case of the Gaussian hypergeometric function, for $r \in(0,1)$, Legendre's complete elliptic integrals of the first kind are defined by

$$
\mathcal{K}(r)=\int_{0}^{\pi / 2}\left(1-r^{2} \sin ^{2} \theta\right)^{-1 / 2} d \theta=\frac{\pi}{2} F\left(\frac{1}{2}, \frac{1}{2} ; 1 ; r^{2}\right) .
$$

2010 AMS Mathematics subject classification. Primary 33C05.

Keywords and phrases. Gaussian hypergeometric function, Ramanujan's cubic transformation, inequality.

This research was supported by the Natural Science Foundation of China (grant Nos. 11371125, 11401191 and 61374086) and the Natural Science Foundation of Zhejiang Province (grant No. LY13A010004).

Received by the editors on October 15, 2012, and in revised form on June 4, 2014. 
Some of the most important properties of the elliptic integrals $\mathcal{K}(r)$ are the Landen identities:

$$
\begin{aligned}
& \mathcal{K}\left(\frac{2 \sqrt{r}}{1+r}\right)=(1+r) \mathcal{K}(r), \\
& \mathcal{K}\left(\frac{1-r}{1+r}\right)=\frac{1+r}{2} \mathcal{K}\left(\sqrt{1-r^{2}}\right),
\end{aligned}
$$

namely,

$$
\begin{aligned}
F\left(\frac{1}{2}, \frac{1}{2} ; 1 ; \frac{4 r}{(1+r)^{2}}\right) & =(1+r) F\left(\frac{1}{2}, \frac{1}{2} ; 1 ; r^{2}\right) \\
F\left(\frac{1}{2}, \frac{1}{2} ; 1 ;\left(\frac{1-r}{1+r}\right)^{2}\right) & =\frac{1+r}{2} F\left(\frac{1}{2}, \frac{1}{2} ; 1 ; 1-r^{2}\right) .
\end{aligned}
$$

For zero-balanced Gaussian hypergeometric functions $F(a, b ; a+$ $b ; x), a, b>0$, Simić and Vuorinen [10] determined the maximal region of the $a b$ plane where equations (1.2) and (1.3) turn on respective inequalities valid for each $x \in(0,1)$.

As is known to all, Ramanujan's cubic transformation is defined as

$$
\begin{aligned}
F\left(\frac{1}{3}, \frac{2}{3} ; 1 ; 1-\left(\frac{1-r}{1+2 r}\right)^{3}\right) & =(1+2 r) F\left(\frac{1}{3}, \frac{2}{3} ; 1 ; r^{3}\right) \\
F\left(\frac{1}{3}, \frac{2}{3} ; 1 ;\left(\frac{1-r}{1+2 r}\right)^{3}\right) & =\frac{1+2 r}{3} F\left(\frac{1}{3}, \frac{2}{3} ; 1 ; 1-r^{3}\right) .
\end{aligned}
$$

Inspired by the ideas of Simic and Vuorinen [10], we find the maximal region of the $a b$ plane for $F(a, b ; a+b ; x), a, b>0$, where equations (1.4) and (1.5) turn on respective inequalities valid for each $x \in(0,1)$.

The following asymptotic formulas for the zero-balanced hypergeometric function (see $[\mathbf{5}, \mathbf{6}]$ ) will be used in this paper.

$$
F(a, b ; a+b ; r) \sim-\frac{1}{B(a, b)} \log (1-r)
$$

and

$$
\begin{aligned}
B(a, b) F(a, b ; a+b ; r)+\log & (1-r) \\
& =R(a, b)+O((1-r) \log (1-r)),
\end{aligned}
$$


as $r$ tends to 1 , where

$$
B(z, w)=\frac{\Gamma(z) \Gamma(w)}{\Gamma(z+w)}, \quad \operatorname{Re} z>0, \text { Re } w>0
$$

is the classical beta function,

$$
\begin{gathered}
R(a, b)=-\Psi(a)-\Psi(b)-2 \gamma, \quad R\left(\frac{1}{3}, \frac{2}{3}\right)=\log 27 \\
\Psi(z)=\frac{d}{d z}(\log \Gamma(z))=\frac{\Gamma^{\prime}(z)}{\Gamma(z)}, \quad \operatorname{Re} z>0
\end{gathered}
$$

and $\gamma$ is the Euler-Mascheroni constant.

Lemma 1.1. (see [10, Lemma 1.1]). Suppose that the power series

$$
f(x)=\sum_{n=0}^{\infty} a_{n} x^{n}
$$

and

$$
g(x)=\sum_{n=0}^{\infty} b_{n} x^{n}
$$

have the radius of convergence $r>0$ and $a_{n}, b_{n}>0$ for all $n \in$ $\{0,1,2, \ldots\}$. Let $h(x)=f(x) / g(x)$. Then

(i) if the sequence $\left\{a_{n} / b_{n}\right\}_{n=0}^{\infty}$ is (strictly) increasing (decreasing), then $h(x)$ is also (strictly) increasing (decreasing) on $(0, r)$;

(ii) if the sequence $\left\{a_{n} / b_{n}\right\}$ is (strictly) increasing (decreasing) for $0<n \leq n_{0}$ and (strictly) decreasing (increasing) for $n>n_{0}$, then there exists an $x_{0} \in(0, r)$ such that $h(x)$ is (strictly) increasing (decreasing) on $\left(0, x_{0}\right)$ and (strictly) decreasing (increasing) on $\left(x_{0}, r\right)$.

2. Main results. For convenience, we first introduce the following regions in $\left\{(a, b) \in \mathbf{R}^{2} \mid a>0, b>0\right\}$ (see Figure 1):

$$
\begin{aligned}
& D_{1}=\left\{(a, b) \mid a, b>0, a b \leq \frac{2}{9}, a b-\frac{2}{9}(a+b) \leq 0\right\}, \\
& D_{2}=\left\{(a, b) \mid a, b>0, a b<\frac{2}{9}, a b-\frac{2}{9}(a+b)>0\right\},
\end{aligned}
$$




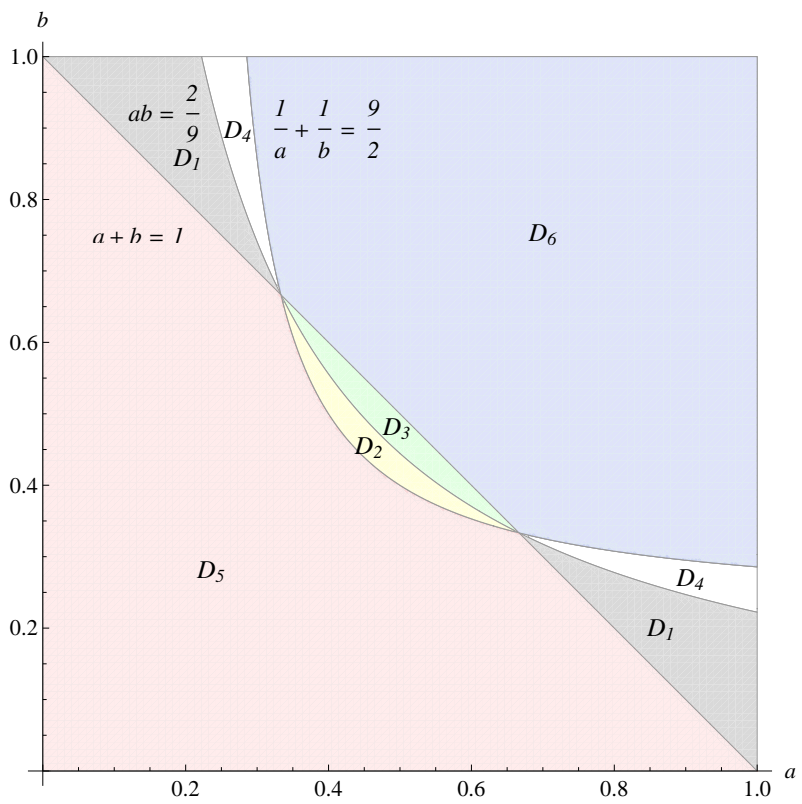

Figure 1 . The regions $D_{i}, i=1,2, \ldots, 6$.

$$
\begin{aligned}
& D_{3}=\left\{(a, b) \mid a, b>0, a b \geq \frac{2}{9}, a b-\frac{2}{9}(a+b) \geq 0\right\}, \\
& D_{4}=\left\{(a, b) \mid a, b>0, a b>\frac{2}{9}, a b-\frac{2}{9}(a+b)<0\right\}, \\
& D_{5}=\left\{(a, b) \mid a, b>0, a+b \leq 1, a b-\frac{2}{9}(a+b) \leq 0\right\}, \\
& D_{6}=\left\{(a, b) \mid a, b>0, a+b \geq 1, a b-\frac{2}{9}(a+b) \geq 0\right\} .
\end{aligned}
$$

Clearly, $D_{1} \cup D_{2} \cup D_{3} \cup D_{4}=\left\{(a, b) \in \mathbf{R}^{2} \mid a>0, b>0\right\}, D_{5} \subset D_{1}$ and $D_{6} \subset D_{3}$.

Theorem 2.1. If $(a, b) \in D_{1}$, then the inequality

$$
F\left(a, b ; a+b ; \frac{9 r\left(1+r+r^{2}\right)}{(1+2 r)^{3}}\right) \leq(1+2 r) F\left(a, b ; a+b ; r^{3}\right)
$$


holds for all $r \in(0,1)$. Also, if $(a, b) \in D_{3}$, then the reversed inequality

$$
F\left(a, b ; a+b ; \frac{9 r\left(1+r+r^{2}\right)}{(1+2 r)^{3}}\right) \geq(1+2 r) F\left(a, b ; a+b ; r^{3}\right)
$$

takes place for each $r \in(0,1)$, with equality in each instance if and only if $(a, b)=(1 / 3,2 / 3)$ or $(a, b)=(2 / 3,1 / 3)$.

In the remaining region $(a, b) \in D_{2} \cup D_{4}$, neither of the above inequalities holds for each $r \in(0,1)$.

Theorem 2.2. If $(a, b) \in D_{1}$, then the double inequality

$$
1 \leq \frac{(1+2 r) F\left(a, b ; a+b ; r^{3}\right)}{F\left(a, b ; a+b ; 9 r\left(1+r+r^{2}\right) /(1+2 r)^{3}\right)} \leq \frac{\sqrt{3} B(a, b)}{2 \pi}
$$

holds for all $r \in(0,1)$. And, if $(a, b) \in D_{3}$, then inequality (2.3) is reversed,

$$
\frac{\sqrt{3} B(a, b)}{2 \pi} \leq \frac{(1+2 r) F\left(a, b ; a+b ; r^{3}\right)}{F\left(a, b a+b ; 9 r\left(1+r+r^{2}\right) /(1+2 r)^{3}\right)} \leq 1 .
$$

Moreover, both bounds in inequalities (2.3) and (2.4) are sharp and each equality is reached for $a=1 / 3$ and $b=2 / 3$, or $a=2 / 3$ and $b=1 / 3$.

Corollary 2.3. For $r \in(0,1)$, and $(a, b) \in D_{1}$, one has

$$
\begin{aligned}
\frac{2 \pi}{\sqrt{3}} \frac{1}{B(a, b)} F\left(a, b ; a+b ; r^{3}\right) & <F\left(a, b ; a+b ; \frac{9 r\left(1+r+r^{2}\right)}{(1+2 r)^{3}}\right) \\
& <3 F\left(a, b ; a+b ; r^{3}\right) .
\end{aligned}
$$

In the region $(a, b) \in D_{3}$, one has

$$
\begin{aligned}
F\left(a, b ; a+b ; r^{3}\right) & <F\left(a, b ; a+b ; \frac{9 r\left(1+r+r^{2}\right)}{(1+2 r)^{3}}\right) \\
& <\frac{6 \pi}{\sqrt{3}} \frac{1}{B(a, b)} F\left(a, b ; a+b ; r^{3}\right) .
\end{aligned}
$$

Theorem 2.4. Let $B=B(a, b)$ and $R=R(a, b)$ be defined as in (1.8) and (1.9), respectively. Then for $(a, b) \in D_{5}$, inequality 
(2.7)

$$
\begin{aligned}
0 & \leq(1+2 r) F\left(a, b ; a+b ; r^{3}\right)-F\left(a, b ; a+b ; \frac{9 r\left(1+r+r^{2}\right)}{(1+2 r)^{3}}\right) \\
& \leq \frac{2(R-\log 27)}{B}
\end{aligned}
$$

holds for all $r \in(0,1)$. Also, for $(a, b) \in D_{6}$,

$$
\begin{aligned}
0 & \leq F\left(a, b ; a+b ; \frac{9 r\left(1+r+r^{2}\right)}{(1+2 r)^{3}}\right)-(1+2 r) F\left(a, b ; a+b ; r^{3}\right) \\
& \leq \frac{2(\log 27-R)}{B} .
\end{aligned}
$$

\section{Theorem 2.5.}

(i) For $(a, b) \in D_{1}$ and each $x \in(0,1)$, one has

$$
\frac{1}{3} \leq \frac{F\left(a, b ; a+b ;((1-x) /(1+2 x))^{3}\right)}{(1+2 x) F\left(a, b ; a+b ; 1-x^{3}\right)} \leq \frac{\sqrt{3} B(a, b)}{6 \pi} .
$$

(ii) For $(a, b) \in D_{3}$ and each $x \in(0,1)$, one has

$$
\frac{\sqrt{3} B(a, b)}{6 \pi} \leq \frac{F\left(a, b ; a+b ;((1-x) /(1+2 x))^{3}\right)}{(1+2 x) F\left(a, b ; a+b ; 1-x^{3}\right)} \leq \frac{1}{3} .
$$

(iii) For $(a, b) \in D_{5}$ and each $x \in(0,1)$, we have

(2.11) $(1+2 x) F\left(a, b ; a+b ; 1-x^{3}\right)$

$$
\begin{aligned}
& \leq 3 F\left(a, b ; a+b ;\left(\frac{1-x}{1+2 x}\right)^{3}\right) \\
& \leq(1+2 x)\left[F\left(a, b ; a+b ; 1-x^{3}\right)+\frac{2(R(a, b)-\log 27)}{B(a, b)}\right] .
\end{aligned}
$$

(iv) For $(a, b) \in D_{6}$ and each $x \in(0,1)$, we have

$$
\begin{aligned}
0 \leq & (1+2 x) F\left(a, b ; a+b ; 1-x^{3}\right) \\
& -3 F\left(a, b ; a+b ;\left(\frac{1-x}{1+2 x}\right)^{3}\right) \\
\leq & \frac{2(1+2 x)(\log 27-R(a, b))}{B(a, b)} .
\end{aligned}
$$


3. Proofs of theorems. In order to prove our main results, we introduce several symbols. Throughout this section, we let

$$
F(x)=F(a, b ; a+b ; x), \quad G(x)=F(a, b ; a+b+1 ; x),
$$

where $a, b>0$ with $(a, b) \neq(1 / 3,2 / 3)$ and $(a, b) \neq(2 / 3,1 / 3)$, and

$$
F^{*}(x)=F\left(\frac{1}{3}, \frac{2}{3} ; 1 ; x\right), \quad G^{*}(x)=F\left(\frac{1}{3}, \frac{2}{3} ; 2 ; x\right) .
$$

\section{Lemma 3.1.}

(i) The function $f(r)=F(r) / F^{*}(r)$ is strictly decreasing in $(0,1)$ on $D_{1}$, and strictly increasing in $(0,1)$ on $D_{3}$. Moreover, if $(a, b) \in$ $D_{2}\left(D_{3}\right.$, respectively), then there exists $r_{0}\left(r_{0}^{*}\right.$, respectively) such that $f(r)$ is strictly increasing (decreasing, respectively) in $\left(0, r_{0}\right)\left(\left(0, r_{0}^{*}\right)\right.$, respectively $)$, and strictly decreasing (increasing, respectively) in $\left(r_{0}, 1\right)\left(\left(r_{0}^{*}, 1\right)\right.$, respectively $)$.

(ii) The function $g(r)=G(r) / G^{*}(r)$ is strictly decreasing in $(0,1)$ on $D_{5}$ and strictly increasing in $(0,1)$ on $D_{6}$.

Proof. For part (i), denote by $A_{n}=(a, n)(b, n) /[(a+b, n) n !]$ and $A_{n}^{*}=(1 / 3, n)(2 / 3, n) /[(n) !]^{2}$, then

$$
f(r)=\frac{F(r)}{F^{*}(r)}=\frac{\sum_{n=0}^{\infty} A_{n} r^{n}}{\sum_{n=0} A_{n}^{*} r^{n}} .
$$

Note that the monotonicity of $\left\{A_{n} / A_{n}^{*}\right\}$ depends on the sign of

$$
H_{n}=\left(a b-\frac{2}{9}\right) n+a b-\frac{2}{9}(a+b) .
$$

We divide the proof into four cases.

Case 1. $(a, b) \in D_{1}$. Then equation (3.2) implies $H_{n}<0$ for $n=0$, $1,2, \ldots$, and $f(r)$ is strictly decreasing on $(0,1)$ by equation $(3.1)$ and Lemma 1.1.

Case 2. $(a, b) \in D_{3}$. Then equation (3.2) implies $H_{n}>0$ for $n=0$, $1,2, \ldots$, and $f(r)$ is strictly increasing on $(0,1)$ by equation $(3.1)$ and Lemma 1.1. 
Case 3. $(a, b) \in D_{2}$. Then from equation (3.2) we conclude that the sequence $\left\{A_{n} / A_{n}^{*}\right\}$ is increasing and then decreasing. By equation (3.1) and Lemma 1.1 (ii), there exists $r_{0} \in(0,1)$ such that $f(r)$ is strictly increasing on $\left(0, r_{0}\right)$ and strictly decreasing on $\left(r_{0}, 1\right)$.

Case 4. $(a, b) \in D_{4}$. Then from equation (3.2) we know that the sequence $\left\{A_{n} / A_{n}^{*}\right\}$ is decreasing and then increasing. By equation (3.1) and Lemma 1.1 (ii), there exists $r_{0}^{*} \in(0,1)$ such that $f(r)$ is strictly decreasing on $\left(0, r_{0}^{*}\right)$ and strictly increasing on $\left(r_{0}^{*}, 1\right)$.

For part (ii), denote $B_{n}=(a, n)(b, n) /[(a+b+1, n) n !]$ and $B_{n}^{*}=$ $(1 / 3, n)(2 / 3, n) /[(2, n)(n) !]$. Then

$$
g(r)=\frac{G(r)}{G^{*}(r)}=\frac{\sum_{n=0}^{\infty} B_{n} r^{n}}{\sum_{n=0} B_{n}^{*} r^{n}} .
$$

Note that the monotonicity of $\left\{B_{n} / B_{n}^{*}\right\}$ depends on the sign of

$$
H_{n}^{*}=\left(a+b+a b-\frac{11}{9}\right) n+\frac{2}{9}(9 a b-a-b-1) \text {. }
$$

We divide the proof into two cases.

Case A. $(a, b) \in D_{5}$. Then $a+b+a b-11 / 9 \leq 11(a+b) / 9-11 / 9 \leq 0$ and $9 a b-a-b-1=9 a b-2(a+b)+(a+b)-1 \leq 0$. Thus, $H_{n}^{*}<0$ for $n=0,1,2, \ldots$ (because $(a, b) \neq(1 / 3,2 / 3)$ and $(a, b) \neq(2 / 3,1 / 3))$ by equation (3.4). Therefore, $g(r)$ is strictly decreasing in $(0,1)$ by equation (3.3) and Lemma 1.1 (i).

Case B. $(a, b) \in D_{6}$. Then $a+b+a b-11 / 9 \geq 11(a+b) / 9-11 / 9 \geq 0$ and $9 a b-a-b-1=9 a b-2(a+b)+(a+b)-1 \geq 0$. Thus, $H_{n}^{*}>0$ for $n=0,1,2, \ldots$ by equation (3.4). Therefore, $g(r)$ is strictly increasing in $(0,1)$ by equation $(3.3)$ and Lemma 1.1 (i).

Proof of Theorem 2.1. Let $x=x(r)=r^{3}$ and $y=y(r)=9 r(1+$ $\left.r+r^{2}\right) /(1+2 r)^{3}$. Then simple computation leads to $0<x<y<1$ for $0<r<1$. Using Lemma 3.1 (i), we get $f(x)>f(y)$ on $D_{1}$, and $f(x)<f(y)$ on $D_{3}$.

For $(a, b) \in D_{1}$, by equation (1.4), one has

$$
\frac{F\left(r^{3}\right)}{F^{*}\left(r^{3}\right)}>\frac{F(y)}{F^{*}(y)}
$$




$$
F(y)<\frac{F^{*}(y)}{F^{*}\left(r^{3}\right)} F\left(r^{3}\right)=(1+2 r) F\left(r^{3}\right) .
$$

Thus, equation (2.1) follows.

Inequality (2.2) is obtained analogously. The remaining conclusions easily follow from Lemma 3.1 (i).

Proof of Theorem 2.2. Let $f(r)$ be defined as in Lemma 3.1 (i), then $f(r)$ is strictly decreasing on $D_{1}$. Then asymptotic formula (1.6) leads to

$$
\begin{aligned}
1 & =\lim _{r \rightarrow 0^{+}} \frac{F(r)}{F^{*}(r)}>\frac{F(r)}{F^{*}(r)}>\lim _{r \rightarrow 1^{-}} \frac{F(r)}{F^{*}(r)} \\
& =\frac{B(1 / 3,2 / 3)}{B(a, b)}=\frac{2 \sqrt{3} \pi}{3 B(a, b)}
\end{aligned}
$$

and

$$
\frac{\sqrt{3} B(a, b)}{2 \pi} \frac{1}{F^{*}(y(r))}>\frac{1}{F(y(r))} \Longrightarrow \frac{\sqrt{3} B(a, b)}{2 \pi} \frac{F^{*}(x(r))}{F^{*}(y(r))}>\frac{F(x(r))}{F(y(r))} .
$$

Thus, inequality (2.3) is clear.

Inequality (2.4) valid on $D_{3}$ can be proved similarly.

Lemma 3.2. The function

$$
\begin{aligned}
J(r)= & \left(1+2 r^{1 / 3}\right) F(a, b ; a+b ; r) \\
& -F\left(a, b ; a+b ; \frac{9 r^{1 / 3}\left(1+r^{1 / 3}+r^{2 / 3}\right)}{\left(1+2 r^{1 / 3}\right)^{3}}\right)
\end{aligned}
$$

is strictly increasing in $(0,1)$ on $D_{5}$ and strictly decreasing in $(0,1)$ on $D_{6}$.

Proof. Let $z=9 r^{1 / 3}\left(1+r^{1 / 3}+r^{2 / 3}\right) /\left(1+2 r^{1 / 3}\right)^{3}$. Then

$$
1-z=\frac{\left(1-r^{1 / 3}\right)^{3}}{\left(1+2 r^{1 / 3}\right)^{3}}, \quad \frac{d z}{d r}=\frac{3\left(1-r^{1 / 3}\right)^{2}}{r^{2 / 3}\left(1+2 r^{1 / 3}\right)^{4}} .
$$

Note that

$$
(1-x) F(a+1, b+1 ; a+b+1 ; x)=F(a, b ; a+b+1 ; x) .
$$


Differentiating $J(r)$ gives

$$
\begin{aligned}
& r^{2 / 3}\left(1-r^{1 / 3}\right) J^{\prime}(r)=\frac{2}{3}\left(1-r^{1 / 3}\right) F(a, b ; a+b ; r) \\
& \quad+\frac{a b}{a+b} \frac{r^{2 / 3}\left(1+2 r^{1 / 3}\right)\left(1-r^{1 / 3}\right)}{1-r} \\
& \quad \times F(a, b ; a+b+1 ; r)-\frac{3 a b}{(a+b)\left(1+2 r^{1 / 3}\right)} F(a, b ; a+b+1 ; z) \\
& =\frac{2}{3}\left(1-r^{1 / 3}\right) F(r)+\frac{a b}{a+b} \frac{r^{2 / 3}\left(1+2 r^{1 / 3}\right)\left(1-r^{1 / 3}\right)}{1-r} G(r) \\
& -\frac{3 a b}{(a+b)\left(1+2 r^{1 / 3}\right)} G(z) .
\end{aligned}
$$

On the other hand, differentiating the Ramanujan cubic transformation, we get

$$
\begin{aligned}
\frac{2}{3} \frac{G^{*}(z)}{1+2 r^{1 / 3}}= & \frac{2}{3}\left(1-r^{1 / 3}\right) F^{*}(r) \\
& +\frac{2}{9} \frac{r^{2 / 3}\left(1+2 r^{1 / 3}\right)\left(1-r^{1 / 3}\right)}{1-r} G^{*}(r) .
\end{aligned}
$$

Let $g(r)$ be defined as in Lemma 3.1 (ii), then $g(r)$ is strictly decreasing in $(0,1)$ on $D_{5}$. Then from $0<r<z<1$ we get $g(r)>g(z)$, namely,

$$
G(z)<\frac{G^{*}(z)}{G^{*}(r)} G(r) .
$$

Equations (3.5) and (3.6) together with inequality (3.7) yield

$$
\begin{aligned}
r^{2 / 3} & \left(1-r^{1 / 3}\right) J^{\prime}(r) \\
> & \frac{2}{3}\left(1-r^{1 / 3}\right) F(r) \\
& \quad+\frac{a b}{a+b} \frac{r^{2 / 3}\left(1+2 r^{1 / 3}\right)\left(1-r^{1 / 3}\right)}{1-r} G(r)-\frac{3 a b}{(a+b)\left(1+2 r^{1 / 3}\right)} \frac{G^{*}(z)}{G^{*}(r)} G(r) \\
= & \frac{2}{3}\left(1-r^{1 / 3}\right) F(r)+\frac{a b}{a+b} \frac{r^{2 / 3}\left(1+2 r^{1 / 3}\right)\left(1-r^{1 / 3}\right)}{1-r} G(r)
\end{aligned}
$$




$$
\begin{aligned}
& -\frac{3 a b}{(a+b)\left(1+2 r^{1 / 3}\right)} \\
& \times\left(\left(1-r^{1 / 3}\right)\left(1+2 r^{1 / 3}\right) \frac{F^{*}(r)}{G^{*}(r)}+\frac{1}{3} \frac{r^{2 / 3}\left(1+2 r^{1 / 3}\right)^{2}\left(1-r^{1 / 3}\right)}{1-r}\right) G(r) \\
= & \frac{2}{3}\left(1-r^{1 / 3}\right) F(r)-\frac{3 a b}{(a+b)}\left(1-r^{1 / 3}\right) \frac{F^{*}(r)}{G^{*}(r)} G(r) \\
= & \frac{2}{3}\left(1-r^{1 / 3}\right)\left[F(r)-\frac{9 a b}{2(a+b)} \frac{F^{*}(r)}{G^{*}(r)} G(r)\right] .
\end{aligned}
$$

Note that

$$
\frac{F^{\prime}(r)}{F^{*^{\prime}}(r)}=\frac{9 a b}{2(a+b)} \frac{G(r)}{G^{*}(r)}
$$

Thus,

$$
\frac{3}{2} r^{2 / 3} J^{\prime}(r)>F(r)-\frac{F^{\prime}(r)}{F^{*^{\prime}}(r)} F^{*}(r)=\frac{F^{2}(r)}{F^{*^{\prime}}(r)}\left(\frac{F^{*}(r)}{F(r)}\right)^{\prime} .
$$

It follows from Lemma 3.1 (i) and $D_{5} \subset D_{1}$ that $\left(F^{*}(r) / F(r)\right)^{\prime} \geq 0$ on $D_{5}$. Hence, $J^{\prime}(r)>0$ and $J(r)$ is strictly increasing in $(0,1)$ on $\bar{D}_{5}$.

Since $g(r)$ is strictly increasing in $(0,1)$ on $D_{6}$, we have $g(z)>g(r)$, namely,

$$
G(z)>\frac{G^{*}(z)}{G^{*}(r)} G(r)
$$

Making use of a similar argument, one has

$$
\frac{3}{2} r^{2 / 3} J^{\prime}(r)<\frac{F^{2}(r)}{F^{*^{\prime}}(r)}\left(\frac{F^{*}(r)}{F(r)}\right)^{\prime}<0,
$$

since $f(r)=F(r) / F^{*}(r)$ is strictly increasing in $(0,1)$ on $D_{6} \subset D_{3}$. Hence, $J(r)$ is strictly decreasing in $(0,1)$ on $D_{6}$.

Proof of Theorem 2.4. From Lemma 3.2, we clearly see that

$$
\lim _{r \rightarrow 0^{+}} J(r)<J(r)<\lim _{r \rightarrow 1^{-}} J(r), \quad \text { on } D_{5},
$$

and

$$
\lim _{r \rightarrow 1^{-}} J(r)<J(r)<\lim _{r \rightarrow 0^{+}} J(r), \quad \text { on } D_{6} .
$$


Clearly, $\lim _{r \rightarrow 0^{+}} J(r)=0$, and, by equation (1.7), we have

$$
\begin{aligned}
\lim _{r \rightarrow 1^{-}} J(r) & =\lim _{r \rightarrow 1^{-}} \frac{3 R(a, b)-3 \log (1-r)-\left(R(a, b)-3 \log \left[\left(1-r^{1 / 3}\right) /\left(1+2 r^{1 / 3}\right)\right]\right)+o(1)}{B(a, b)} \\
& =\frac{2(R(a, b)-\log 27)}{B(a, b)} .
\end{aligned}
$$

The assertion of Theorem 2.4 follows.

Proof of Theorem 2.5. Theorem 2.5 follows from Theorems 2.2 and 2.4 with $x=(1-r) /(1+2 r) \in(0,1)$.

Acknowledgments. The authors would like to thank the anonymous referee for providing a copy of his Mathematica notebook which was very helpful to complete Figure 2.

\section{REFERENCES}

1. M. Abramowitz and I.A. Stegun, eds., Handbook of mathematical functions with formulas, graphs and mathematical tables, Dover, New York, 1965.

2. G.D. Anderson, R.W. Barnard, K.C. Richards, M.K. Vamanamurthy and M. Vuorinen, Inequalities for zero-balanced hypergeometric functions, Trans. Amer. Math. Soc. 347 (1995), 1713-1723.

3. G.D. Anderson, M.K. Vamanamurthy and M. Vuorinen, Conformal invariants, inequalities, and quasiconformal maps, John Wiley \& Sons, New York, 1997.

4. R. Askey, Handbooks of special functions, in A century of mathematics in America Part III, P. Duren. ed., American Mathematical Society, Providence. RI, 1989.

5. B.C. Berndt, Ramanujan's notebooks, Part I, Springer-Verlag, New York, 1985.

6. R.J. Evans, Ramanujan's second notebooks: Asymptotic expansions for hypergeometric series and related functions, Academic Press, Boston, 1988.

7. F.W.J. Olver, D.W. Lozier, R.F. Boisvert and C.W. Clark, eds., NIST Handbook of mathematical functions, Cambridge University Press, Cambridge, 2010.

8. A.P. Prudnikov, Yu.A. Brychkov and O.I. Marichev, Integrals and series, in More special functions, Gordon \& Breach, New York, 1990.

9. S.L. Qiu and M. Vuorinen, Special functions in geometric function theory, in Handbook of complex analysis: Geometric function theory, Elsevier Science, Amsterdam, 2005.

10. S. Simić and M. Vuorinen, Landen inequalities for zero-balanced hypergeometric functions, Abstr. Appl. Anal. (2012), Art. ID. 932061. 
Department of Mathematics, Huzhou Teachers College, Huzhou 313000, P.R. ChinA

Email address: wmk000@126.com

Department of Mathematics, Huzhou Teachers College, Huzhou 313000 , P.R. CHINA

Email address: chuyuming@hutc.zj.cn

College of Mathematics and Econometrics, Hunan University, Changsha 410082, P.R. CHINA

Email address: ypjiang@hnu.edu.cn 